\title{
Self-Coexistence Strategy in Heterogeneous Cognitive Radio Networks
}

\author{
Qiang Zhang1, Yong Zhang1, Mei Song1, Dawei Dang² \\ ${ }^{1}$ Beijing Key Laboratory of Work Safety Intelligent Monitoring, Beijing University of Posts and Telecommunications, Beijing, \\ China \\ ${ }^{2}$ Industry Information Center, China United Network Communications Corporation Qingdao Branch, Qingdao, China \\ Email: zhangqiang1993@foxmail.com, yongzhang@bupt.edu.cn, songm@bupt.edu.cn, dangdawei1@chinaunicom.cn
}

How to cite this paper: Zhang, Q., Zhang, Y., Song, M. and Dang, D.W. (2017) SelfCoexistence Strategy in Heterogeneous Cognitive Radio Networks. Int. J. Communications, Network and System Sciences, 10, 311-323.

https://doi.org/10.4236/ijcns.2017.105B031

Received: June 30, 2017

Accepted: August 1, 2017

Published: August 4, 2017

\begin{abstract}
Different from general cognitive wireless networks, there is no centralized scheduling and management infrastructure among heterogeneous cognitive networks. Multiple cells may operate in the same vicinity resulting in unfair spectrum occupation time (when the cells belong to different industries) and degraded performance of the cellular networks. A distributed self-coexistence mechanism is necessary. In this paper, we take the self-coexistence of multi users in heterogeneous scenarios as the problem of spectrum allocation in non-cooperative mode. Hence we propose Fair Self-Coexistence Strategy (FSCS). In this strategy, not only the fairness of occupation time is considered, but also different competitive priority metric based on Quality of Service (QoS) is adopted. Each cognitive cell independently completes the spectrum allocation process, by use of sensing techniques and perceptual information about neighboring network cells. The simulation experiment results show that our spectrum allocation strategy guarantees the fairness among the heterogeneous secondary networks. And in the resource scarce environment, our strategy can effectively achieve the differentiation competition results.
\end{abstract}

\section{Keywords}

Heterogeneous Cognitive Networks, Self-Coexisting, Fairness, Game Theory

\section{Introduction}

In recent years, with the rapid increase of the wireless network users, the huge demand for the spectrum of the wireless network makes the spectrum resources become increasingly scarce. But the research found that the spectrum resource utilization rate is generally low [1] [2] [3]. To solve this serious problem, the dynamic spectrum access technology came into being. The technology allows 
secondary network with cognitive capability use the idle licensed spectrum (i.e., spectrum holes), to ease the problem of spectrum scarcity. Inevitably, however, a number of cognitive networks located in near region would share the same authorized spectrum [4].

The IEEE 802.22 standard [5] supports wireless broadband access in rural and remote areas using white spaces of the TV spectrum in VHF and UHF bands while avoiding interference with PUs. A typical Cognitive Radio (CR) network based on IEEE802.22 is shown in Figure 1, where the digital TV (DTV) transmitter and TV are licensed users (hence the PUs), and WRAN cells are the SUs. Each WRAN cell houses one BS and several end users, called customer premises equipment (CPE). Due to the large coverage in a typical 802.22 scenario, multiple cells may operate in the same vicinity resulting in unfair spectrum occupation time and degraded performance of the cellular networks.

The coexistence lies in the ability of CRs to sense the frequency spectrum and avoid interference to PUs, which is addressed by the sensing techniques [6]. Self-coexistence, on the other hand, is the ability to ensure interference-free and fair transmissions among the CR cells belongs to different possessors (BS1-3 belongs to possessor 1 and BS2-1 belongs to possessor 2). When multiple cells operate using the same channel, there is a possibility that the CRs will try to act greedily and occupy all the available channel bandwidth all time. As all devices belonging to CR cells will act in the same way, this may lead to co-channel interference among CR cells. Therefore, an efficient channel assignment method and competitive access is needed to intelligently use channels, thus limiting or avoiding unfairness. The self-coexistence of the secondary network has gradually attracted people's attention [7].

However, recent researches mainly focus on the problem of coexistence between primary users (PU) and secondary users (SU) [8], there is little research on the self-coexistence among secondary networks [9]. And the fairness of resource occupancy [10] in the overlaying area is becoming an important problem especially when the heterogeneous networks belong to different possessors. This problem is also proposed by POTEVIO in the TD-LTE230 system.

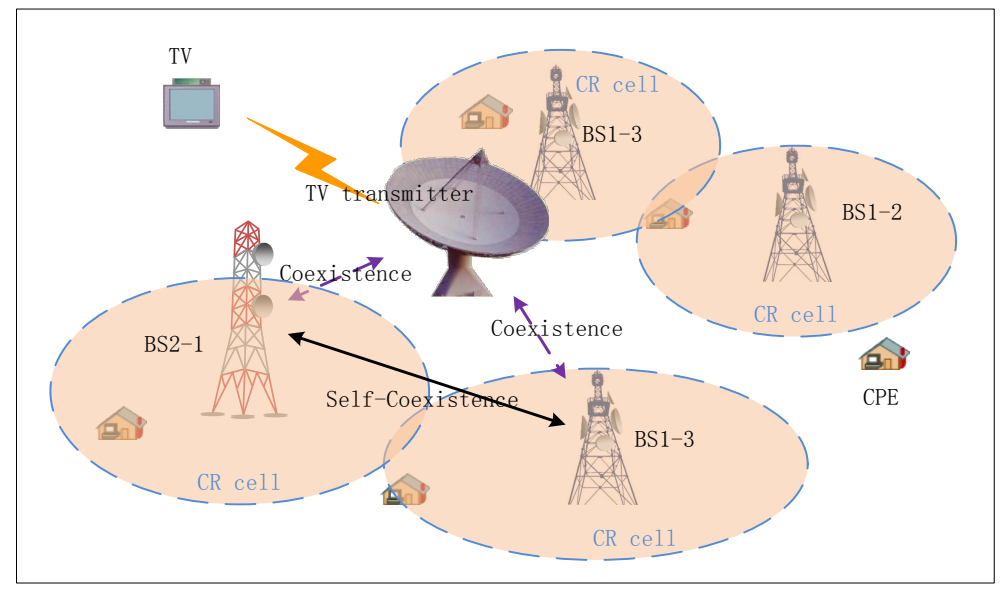

Figure 1. Heterogeneous cognitive networks architecture. 
The main contribution of this paper is the FSCS algorithm, which solve the self-coexistence of heterogeneous networks from two aspects, spectrum allocation and competition.

The rest of the paper is organized as follows. The Section 2 summarizes the related work about the fair self-coexistence mechanism of cognitive networks. The Section 3 defines the system model. The Section 4 describes our channel assignment and competition algorithm. In Section 5, we analyze the results of the simulation. The Section 6 summarizes the significance of the study.

\section{Related Works}

To solve the self-coexistence of heterogeneous cognitive networks, different theoretical models and algorithms have been proposed. Gardellin et al. [11] investigate two kinds of spectrum allocation schemes, namely cooperative and non-cooperative access policies, based on the IEEE 802.22 standard. Gardellin offers us two ways to solve the self-coexistence problem.

Mishra Vishram [12] proposed a graph coloring based fair channel allocation policy for self-coexistence in cognitive radio networks. The proposed scheme allows multiple cognitive radio networks operating over a given region to allocate channels on non-interfering basis with a certain grade of QoS. The scheme allows fair allocation of channels among multiple participating opportunistic networks with varying priorities.

C Guo et al. [13] dedicate to maximizing the sum of fair utility in spectrumsharing networks, where multiple interfering links share one channel, to efficiently trade off system sum-rate and link fairness. And they proposed a power allocation algorithm with fast convergence to a local optimal point.

Sihui Han et al. [14] uncover the cause and effect of variable-width channel coexistence, and develop a MAC-layer scheme, called Fine-grained Spectrum Sharing (FSS), that solves the general problem of fair and efficient spectrum sharing among users with heterogeneous channel-widths.

In 2013, Zhao Yanxiao et al. [9] proposed the Fair MAC Protocol (FMAC). The author establishes the two order Markov state transition model to analyze the performance in heterogeneous network. The goal of Zhao is to improve the fairness index (Index Jain) by adopting the same back-off window. But this protocol has not effective conflict avoidance mechanism, because the same coexistence time window size will lead to high conflict and competition probability of spectrum.

Asif Z. et al. think [15], in the heterogeneous network scenarios, each base station on behalf of a player, the game theory could be used to realize the channel allocation strategy. We take the strategy as a non-cooperative game, which is consistent with the characteristics of the heterogeneous network. The game theory is also used in many kinds of network scenarios, such as wireless mesh networks [16], ad-hoc networks [17], WLAN networks [18], and cognitive network [19].

The achievements above improve the transmission mechanism and efficiency 
of spectrum resources.

\section{System Model}

We consider a set $\mathrm{N} \cup \mathrm{M}$, representing the total number of 802.22 devices where $\mathrm{M}$ is the set of $802.22 \mathrm{BSs}$ and $\mathrm{N}$ is the set of available channels. Each BS represents a WRAN that competes for an unused licensed spectrum with neighboring network cells.

The BSs sense each other's presence as interference. The BSs evaluate the interference using the physical interference model [19] where, as opposed to the protocol model [20], the notion of conflict cannot be modeled as a binary relation by analyzing the mutual interference edge to edge. Specifically, to use the physical interference model we have to compute the minimum value of SINR during the time interval $t$ as in Equation (1).

$$
\operatorname{SINR}_{m}^{n}=\frac{P_{m}^{n}}{\sum_{h \in \mathrm{M}, h \neq m} P_{h}^{n} \cdot X_{h}^{n}+N_{0}}, X_{h}^{n} \in\{0,1\}
$$

Here, $P_{m}^{n}$ represents the received signal power of the SU. According to the free space physical channel transmission model, the received power:

$$
P_{R}=P_{T} G_{T} G_{R} \frac{\lambda}{4 \pi d^{\kappa}}
$$

$\lambda$ is the wavelength of channel $n, d$ is the distance between $t(\mathrm{BS})$ and $R(\mathrm{SU})$. $G_{T}$ is the transmit gain and $G_{R}$ is the receiving gain, $P_{r}$ represents the transmitter power of the BS, $\kappa$ is the attenuation factor. When the cell $h \mathrm{em}$ ploys the same channel $n$, the binary variable $X_{h}^{n}=1$.

We define the global Throughput Matrix:

$$
B=L * C=\left\{b_{m}^{n} \mid b_{m}^{n}=l_{m}^{n} \cdot c_{m}^{n}\right\}
$$

Here, The spectrum resource allocation of cell $\mathrm{m}$ is represented by the spectrum allocation matrix: $L=\left\{l_{m}^{n} \mid l_{m}^{n} \in\{0,1\}\right\}, C_{m}^{n}$ is the channel capacity of channel $n$.

The global throughput can be obtained from the sum of the matrix $B$ :

$$
\text { Throughput }_{\text {tot }}=\sum_{m, n=1}^{M, N} b_{m}^{n}
$$

To get the fairness index, the metric RR of channel $\mathrm{n}$ is defined:

$$
R R_{n}=\left(\sum_{m=1}^{M} T_{m}^{n}\right)^{2} / \sum_{m=1}^{M}\left(T_{m}^{n}\right)^{2} \bullet M
$$

We hope that every cognitive cell has the same occupation time of spectrum resource. That is, we can optimize the target fairness index as follows:

$$
\theta=\frac{1}{C} \cdot \sum_{n=1}^{C} R R_{n}
$$

In Equation (5) and Equation (6) $C$ represents the total number of authorized channels in the current working environment, $T_{m}^{n}$ shows that the time metric 
of channel $n$ is occupied by $m$ cells (BS). As mentioned earlier, $n$ represents the available number of authorized channels detected by the cell $\mathrm{m}$, and subject to $N \leq C$ obviously.

The FSCS algorithm aims to maximize the fairness factor $\theta$ as well as meet the discrepant competition of different services.

\section{Fair Self-Coexisting Strategy}

FSCS is designed based on the four-state smart sensing model, as shown in Figure 2, to achieve fairness among multiple co-existing CRNs.

In a MAC protocol, fairness refers to the ability of an SU from one CRN to share a common channel with multiple users of other CRNs (here after multiple users mean that they are from different networks unless otherwise indicated).

The IEEE 802.22 Standard belongs to the class of centralized CR networks where the SUs are infrastructure oriented and they do not have to create any harmful interference to the PUs. In CR networks when a spectrum opportunity is identified, it is mapped onto a logical channel, which represents a unit of channel assignment.

A BS manages the activities within its WRAN cell:

- Senses the frequency spectrum

- Instructs CPE to perform sensing measurements

- Regulates data transmission

- Makes operational decisions based on its measurements and feedback received from the end users

The IEEE 802.22 standard also addresses the issue of discovering available channels and introduces the concept of the cognitive plane. The cognitive plane includes features and components that:

- Observe the radio spectrum and report the results of its observations

- Determine the location of PUs and SUs in the area

- Maintain spectrum availability information and make decisions regarding channel selection, channel management, and self-coexistence mechanisms

- Enhance the security of the CR-based access

For example, if co-channel interference with other WRAN cells is detected, the cognitive plane takes appropriate action to resolve the fundamental self-

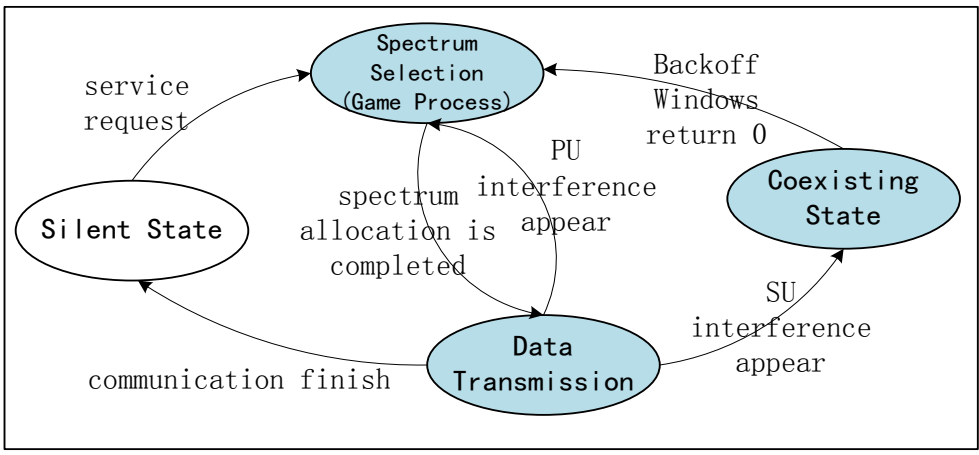

Figure 2. The status transition diagram in secondary network. 
coexistence decision to switch channels.

Besides sensing operations, the self-coexistence mechanism specified by the IEEE 802.22 standard is based on the self-coexistence window (SCW) and the Coexistence Beacon Protocol (CBP), which is a communication protocol implementing beacon transmissions among WRAN cells [21].

\subsection{Distributed Strategies of Channel Selection}

It is well known that cooperation among devices can improve performance in cellular wireless networks. However, ensuring cooperation among CRN cells is difficult, especially in distributed environments where CRN cells can easily deviate to seek more benefits for themselves. Therefore, incentives are needed for cells to behave cooperatively.

In our non-cooperative method, CRN cells distributively make decisions for their own good. As part of a channel assignment, the cells try to ensure that they get the best deal without taking into consideration other cells, thus leading to transmission on the same channel. The utility function $U_{m}$ characterizes the preference of a user for a particular channel. Therefore the performance of the channel assignment algorithm depends significantly on the utility function chosen. We consider the utility functions to maximize the spatial reuse of the spectrum and minimize the interference. The utility of cell $m$ is represented as:

$$
\begin{aligned}
& U_{m}^{n}(t)=\mu \bullet\left\|T_{m}^{n}(t)-\frac{1}{M} \cdot \sum_{h=1}^{M} T_{h}^{n}(t)\right\|_{2}+v \bullet \frac{k^{*} f(m, n)}{C_{m}^{n}} \\
& \left(\begin{array}{l}
\mu+v=1, \mu>0, v>0 \\
k \text { represent data packet size } \\
f(m, n) \text { represent flow present on } \\
\text { link } n
\end{array}\right)
\end{aligned}
$$

In Equation (7) the fairness and congestion avoidance is considered at the same time. $\frac{k^{*} f(m, n)}{C_{m}^{n}}$ reflects the load experienced by all CRNs in shared channel $n$ and satisfy $k^{*} f(m, n) \leq C_{m}^{n}$. Each CRN chooses $\mu$ or $v$ according to the level of congestion in the environment.

We can also classify our multi-player non-cooperative repeated game as a potential game [22]. Classifying our game as a potential game guarantees the convergence to a pure strategy NE solution as proved in [23].

From the perspective of fairness and throughput, we tend to choose $\operatorname{Max}\left(\Delta U_{m}^{n}\right), \Delta U_{m}^{n}=U_{m}^{n}(t+1)-U_{m}^{n}(t)$. If the allowing traffic of channel $\mathrm{n}$ is represented as $W_{m}^{n}(t+1)$, the allowing flow is estimated as:

$$
f(m, n, t+1)=\frac{W_{m}^{n}(t+1)}{k}
$$

The time of channel $n$ would be occupied by new business can be estimated as $\overline{T_{m}^{n}}(t+1)=T_{m}^{n}(t)+\Delta t$. Then we get the priority of channel $n$ :

$$
\sigma_{m}^{n}(t)=U_{m}^{n}(t+1)-U_{m}^{n}(t)
$$

The utility function in Equation (9) expresses the novel channel assignment 
objective which is to maximize the fairness and minimize the congestion. Therefore, the BS $m$ would choose $\xi_{m}=\arg \max \left(\sigma_{m}^{n}\right)$ from set $n=\{1 \cdots N\}$ as the optimal strategy. Where Equation (9) includes the following two aspects:

The revenue of fairness:

$$
\left\|T_{m}^{n}(t)-\frac{1}{M} \cdot \sum_{h=1}^{M} T_{h}^{n}(t)\right\|_{2}-\left\|\overline{T_{m}^{n}}(t+1)-\frac{1}{M} \cdot \sum_{h=1}^{M} T_{h}^{n}(t)\right\|_{2}
$$

The revenue of congestion avoidance:

$$
\frac{k^{*}(f(m, n)-f(m, n, t+1))}{C_{m}^{n}}
$$

Players should play in an almost sequential order, i.e. only one WRAN can changes its strategy in each stage. Similar to the IEEE 802.22, we take backoff window (BW) to solve this problem [24]. As shown in the following section. Besides, the power of SU should be adjusted to achieve spectrum sharing [25].

\subsection{Competition Mechanism}

At each stage $t, B S \mathrm{~m} \in \mathrm{M}$ chooses a channel $\mathrm{n} \in \mathrm{N}$ and a BW (backoff window). We introduce a novel way to compute the $\mathrm{BW}$ (the $\mathrm{BW}$ request window is for CPEs to request upstream bandwidth allocation from the BS), where BW is proportional to the class of service and depends on the number of WRANs that each player senses. Thus:

$$
B W_{m}^{n}=\left\{\begin{array}{c}
\text { if } S U \text { is free of int erference: } \\
{\left[0, \ln \left(Q o S_{-} \text {Metric }_{m}^{n}\right)\right]} \\
\quad \text { otherwise: } \\
{\left[\ln \left(Q o S_{-} \text {Metric }_{m}^{n}\right), Q o S_{-} \text {Metric }_{m}^{n}-1\right]}
\end{array}\right.
$$

Where priority of cell $\mathrm{m}$ :

$$
\begin{gathered}
\text { QoS } S_{-} \text {Metric }_{m}^{n}=v_{m} \cdot \frac{W_{m}^{n}}{\sum_{h=1}^{M} W_{h}^{n} \cdot x_{h}}, h \neq m \\
x_{h}=\left\{\begin{array}{cc}
1 & C P E S(\text { cell } h) \text { share the } 3 \text { ame } \\
& \text { channel with cell } m \\
0 & \text { otherwise }
\end{array}\right.
\end{gathered}
$$

The 3GPP (the 3rd Generation Partnership Project) classifies the services into four priorities (Session Service, Interactive Service, Streaming media service and Background Service). $v_{m}$ represents the priority levels of different services. A BS experiencing a high interference level will try to change its channel more often. The backoff counter is chosen randomly between 0 and QoS_Metric ${ }_{m}^{n}$, and is decreased by one at each stage. When the backoff counter reaches zero, the BS senses the environment and applies the reallocation of its radio. After it changes the channel assignment, the backoff window and the counter are reset as described earlier. We can conclude that using the backoff mechanism, the players play a game in an almost sequential order. The SCW (self-coexisting windows) carry information about the transmitter's ongoing service flows with the BS, also 
about the 802.22 cell as a whole. Thus BSs are capable of implementing mechanisms that allow inter-cell coexistence based on interference and services. From Equation (12) and Equation (13), we can also get that, the higher the priority level, the shorter the coexisting time (to avoid interference) the CRN would fall into.

\section{Simulation Results and Analysis}

We have analyze the performance of the FSCS algorithm in this section. The MATLAB program is driven by the services request and uses object oriented techniques. We abstract the BS, SU and the physical environment as three classes. The simulation adopts the physical channel model, and the attenuation factor $\kappa=2$. The covering radius of cognitive BS is about $\mathcal{R}=20 \mathrm{~km}$, the geographical coverage area is $\Gamma=20 \times 20 \mathrm{~km}^{2}$. The BS, SU and PU are randomly distributed. The number of base stations $(\mathcal{M})$ and secondary users $(\mathbb{S})$ is changeable. Figure 3 shows the network topology when $\mathcal{M}=4, \mathbb{S}=4$ (one SU each BS).

The range of the system operating frequency is $195 \mathrm{MHz}-219 \mathrm{MHz}$, the working bandwidth is $7.5 \mathrm{MHz} / 8 \mathrm{MHz}$, the typical value of $-173 \mathrm{dbm} / \mathrm{Hz}$ is used to denote gauss white noise, the maximum transmit power $\mathrm{P}=29.03 \mathrm{dbW}$. The business request subject to Poisson distribution. Similar to Asif Zeeshan [15] and Yanxiao Zhao [9], we take the Max-Throughput scheme and FMAC scheme to compare with FSCS.

\subsection{Comparison of Fair Index}

The frequency of business requests follows with the Poisson distribution whose $1 / \lambda=50 \mathrm{~min}$, and the fair index of the starting moment is generated randomly. We get the resource occupancy rate as shown in Figure 4.

In Figure 4, the line with plus is the FSCS (when $\mu=0.8$ ), it can be seen that the improvement of fairness in FSCS is very obvious. On the other hand, Zee

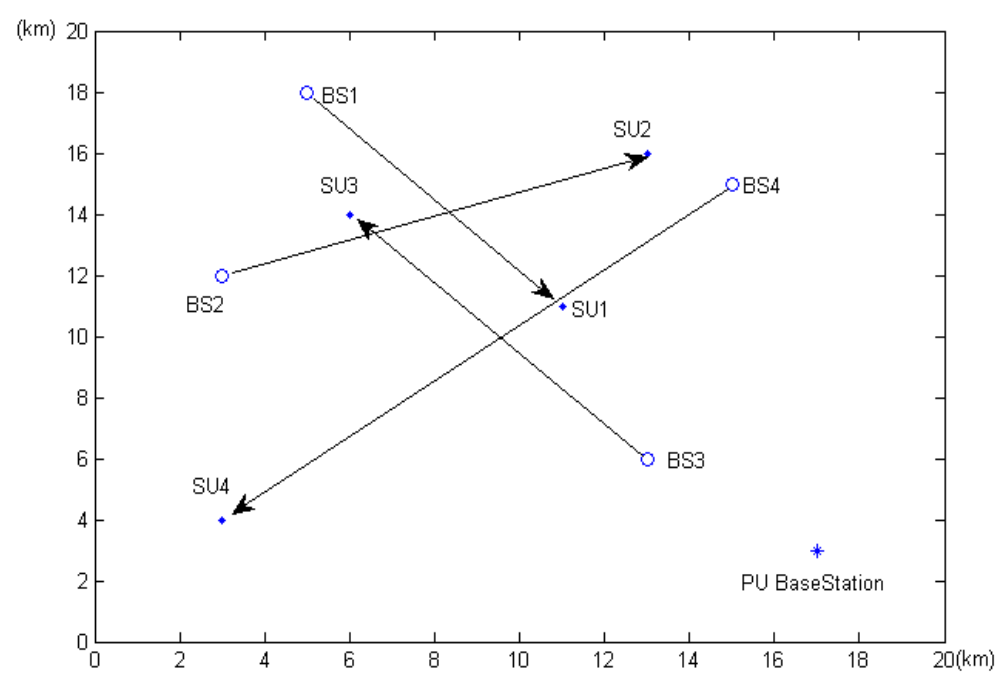

Figure 3. Network topology when 4 BS, 4 SU. 


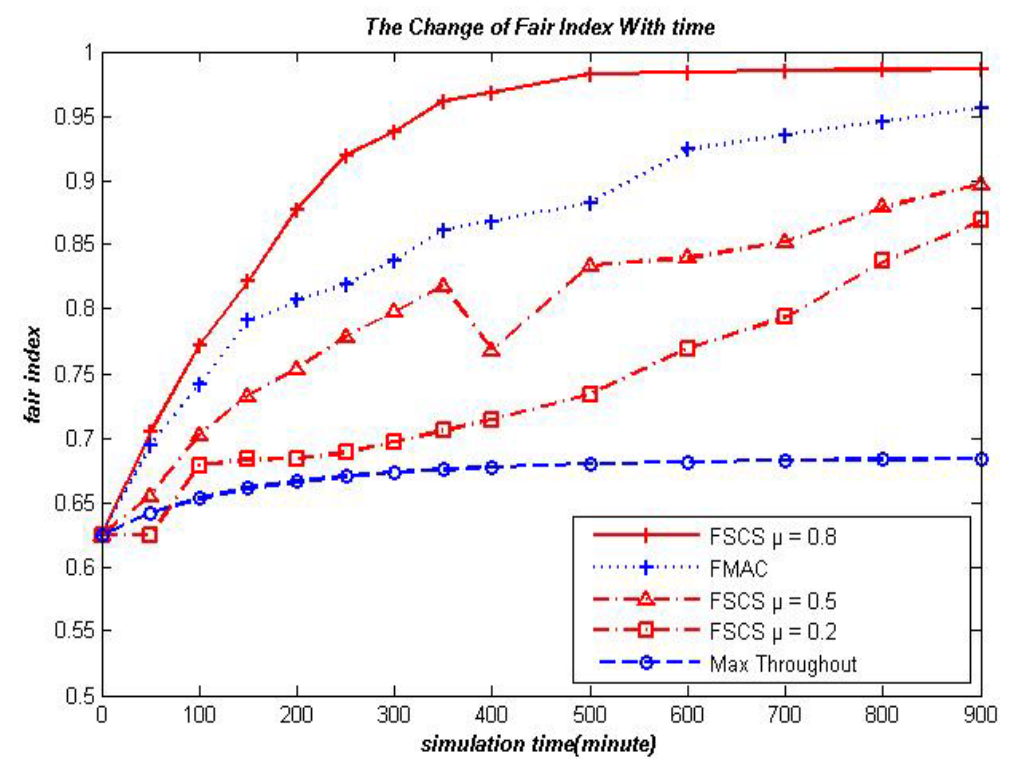

Figure 4. The improvement of fair index.

shan's strategy has a little effect on the improvement of the fair index. We compare the Fair-MAC algorithm [9] with FSCS. We can also get that the convergence time is about 600 minutes.

If the system resources cannot meet the service requests, the mechanism of competition would begin to work. We can observe the different between Figure 4 and Figure 5.

The line with square is the starting value of fair index in the experiment, the line with circle is the scheduling strategy of Zeeshan's, the line with plus is the strategy of this paper $(\mu=0.8)$. It can be seen that, in the case of resource shortage, the strategies are obviously restricted in the promotion of fair index, but the performance of FSCS is still relatively better, because of congestion avoidance and spectrum sharing.

\subsection{Delay Distribution}

Mentioned above, the competitive mechanism is aimed at the optimization of delay performance for the services with high delay priority. We can infer the BSs would start competition procedure from $\mathcal{M}=6$. The result is shown as Figure 6: In the Figure 6, the line with plus is FSCS strategy, and the line with circle is Zeeshan's strategy. As we can see in Figure 6, FSCS algorithm has significant difference delay distribution. In different competitive environment, the strategy can ensure that high priority business would always be implemented first. Zeeshan do not differentiate the types of business, thus the delay distribution is uniform. When $\mathbb{S} \leq 4$, the delay of service time is 0 because of the adequate resource.

\subsection{The Comparison of Throughput}

Zeeshan's strategy aims at the maximized system throughput. So we could fore- 


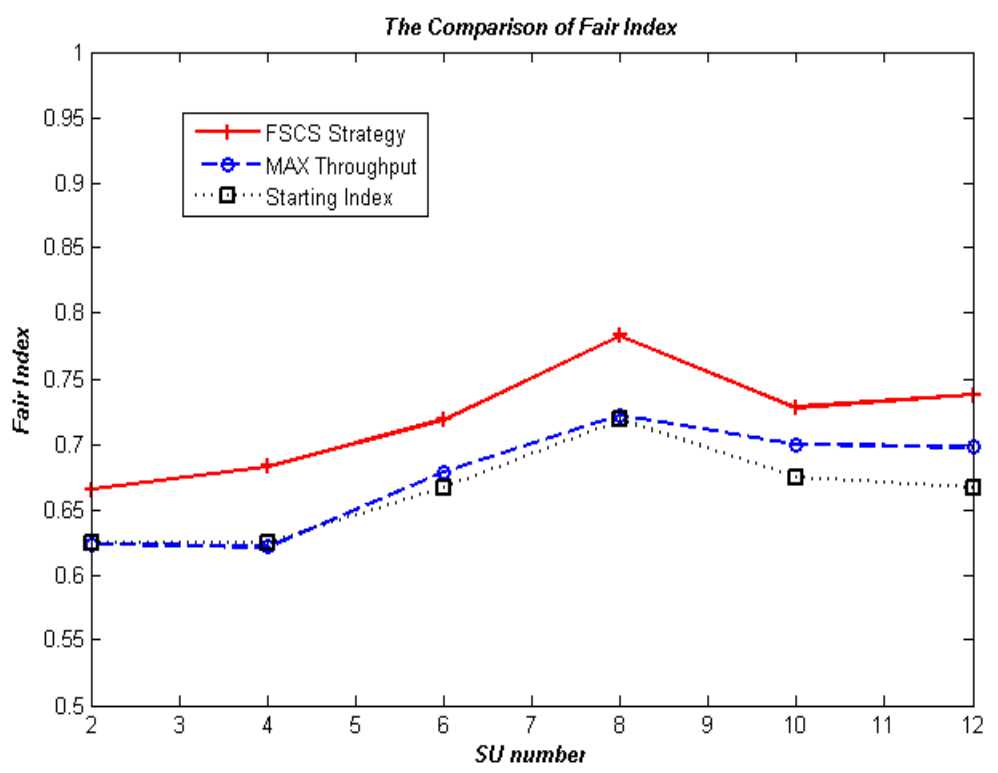

Figure 5. The comparison of fair index in the scarce resources environment.

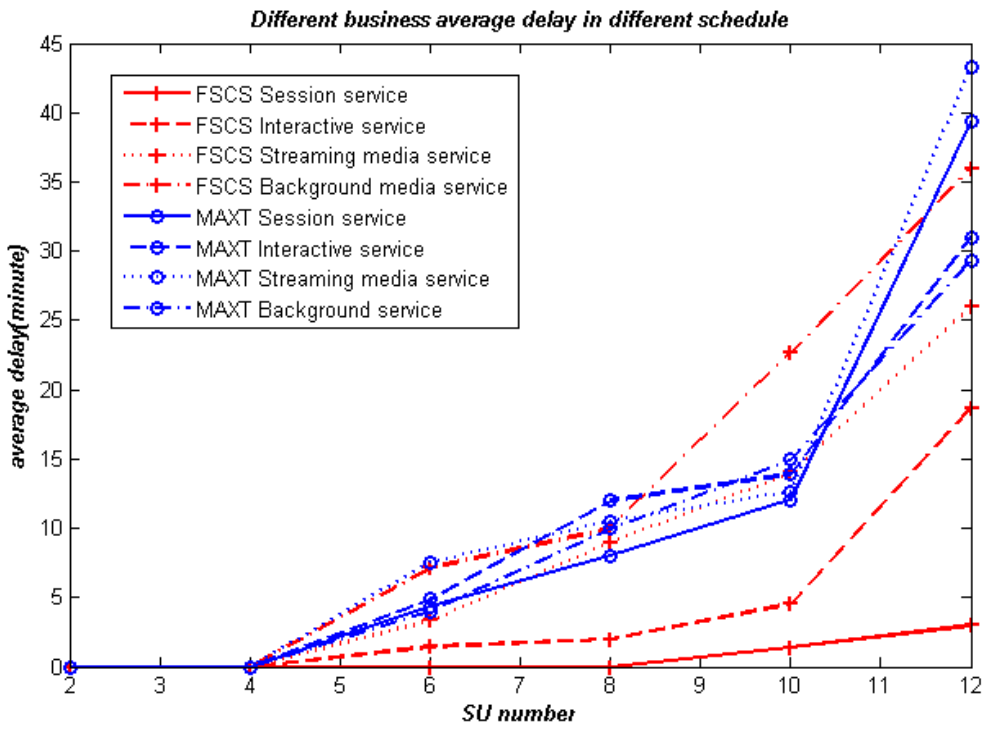

Figure 6. Differentiation delay of different business.

see the difference in throughput performance. Simulation results are shown in Figure 7.

As we can see from the graph, the average throughput of Zeeshan's is better than this paper, especially in the case of sufficient channels (when $\mathbb{S}<5$ ). But in the case of just enough resource $(\mathbb{N}=4)$, the performance of two strategies is almost the same. That is because there is only one channel can be chosen, and that all the scheduling policies play no role, and the allocation of the channels is determined by the environment.

\section{Conclusion}

Based on IEEE 802.22 protocol, this paper solves the problem of fair self-coexis- 


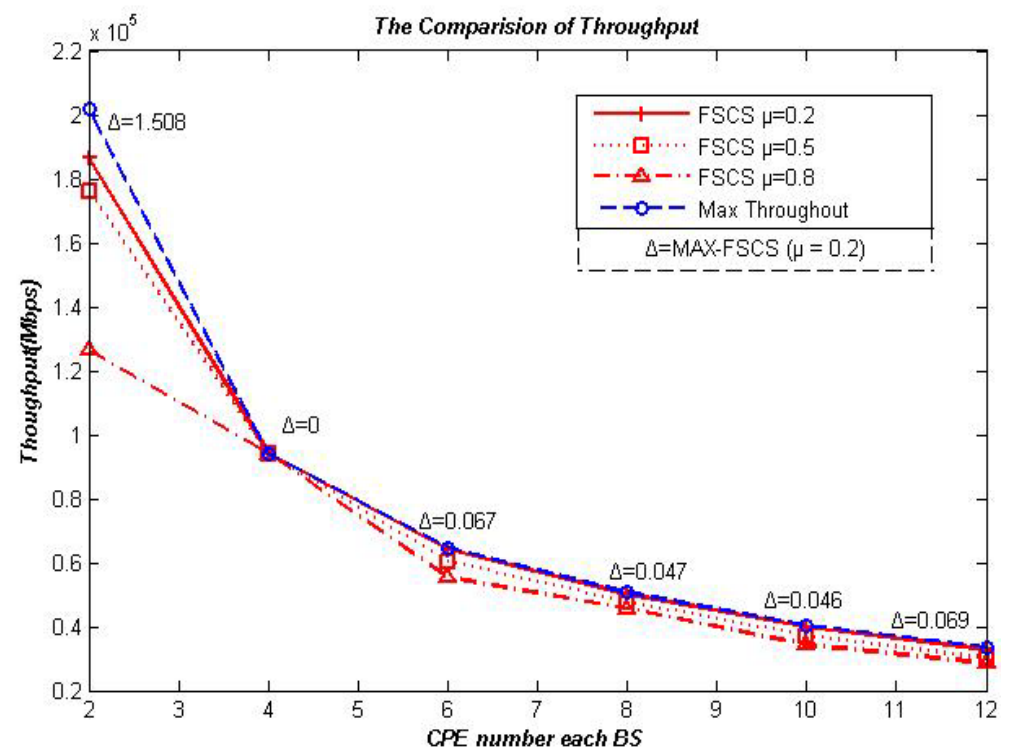

Figure 7. The comparison of average throughput.

tence in heterogeneous cognitive networks. We design the spectrum allocation strategy based on fair index, which ensures that one channel would not be occupied by a cognitive network unfair. Based on the design of backoff window, this paper achieves the differentiation response of different services. In addition, we take other fair coexistence strategy as a comparison, which shows that our strategy improves the fairness of resource occupancy as well as system throughput. Besides, some interesting extensions of this work may include studying the shared algorithm based on different network load, and multi-target spectrum assignment algorithm.

\section{Acknowledgments}

The authors would like to express their thanks to the reviewers for their detailed suggestions. This work was supported by the National Natural Science Foundation of China (61372117).

\section{References}

[1] Han, Z. and Liu, K.J.R. (2008) Resource Allocation for Wireless Networks: Basics, Techniques, and Applications. Cambridge University Press, Cambridge. https://doi.org/10.1017/CBO9780511619748

[2] Mchenry, M.A., et al. (2006) Chicago Spectrum Occupancy Measurements \& Analysis and a Long-Term Studies Proposal. Proceedings of the First International Workshop on Technology, 1. https://doi.org/10.1145/1234388.1234389

[3] Nie, N. and Comaniciu, C. (2005) Adaptive Channel Allocation Spectrum Etiquette for Cognitive Radio Networks. IEEE International Symposium on New Frontiers in Dynamic Spectrum Access Networks, 269-278. https://doi.org/10.1109/DYSPAN.2005.1542643

[4] Ghosh, C., Roy, S. and Cavalcanti, D. (2011) Coexistence Challenges for Heterogeneous Cognitive Wireless Networks in TV White Spaces. IEEE Wireless Communications, 18, 22-31. https://doi.org/10.1109/MWC.2011.5999761 
[5] Wang, S.W., Huang, F. and Wang, C. (2013) Adaptive Proportional Fairness Resource Allocation for OFDM-Based Cognitive Radio Networks. Wireless Networks, 19, 273-284. https://doi.org/10.1007/s11276-012-0465-9

[6] Yucek, T. and Arslan, H. (2009) A Survey of Spectrum Sensing Algorithms for Cognitive Radio Applications. IEEE Communications Surveys \& Tutorials, 11, 116-130. https://doi.org/10.1109/SURV.2009.090109

[7] Han, S., et al. (2014) A Proportional Fair Spectrum Allocation for Wireless Heterogeneous Networks. International Symposium on Consumer Electronics, 1-2.

[8] Haykin, S. (2005) Cognitive Radio: Brain-Empowered Wireless Communications. IEEE Journal on Selected Areas in Communications, 23, 201-220. https://doi.org/10.1109/JSAC.2004.839380

[9] Hortigüela-Martín, V.A., et al. (2013) FMAC: A Fair MAC Protocol for Coexisting Cognitive Radio Networks. Proceedings of IEEE INFOCOM, 12, 1474-1482.

[10] Guan, Z. and Melodia, T. (2016) CU-LTE: Spectrally-Efficient and Fair Coexistence between LTE and Wi-Fi in Unlicensed Bands. The 35 th Annual IEEE International Conference on Computer Communications, San Francisco, 10-14 April 2016, 1-9. https://doi.org/10.1109/INFOCOM.2016.7524589

[11] Gardellin, V., Das, S.K. and Lenzini, L. (2013) Self-Coexistence in Cellular Cognitive Radio Networks Based on the IEEE 802.22 Standard. IEEE Wireless Communications, 20, 52-59. https://doi.org/10.1109/MWC.2013.6507394

[12] Vishram, M., Tong, L.C. and Syin, C. (2014) List Multi-Coloring Based Fair Channel Allocation Policy for Self Coexistence in Cognitive Radio Networks with QoS Provisioning. IEEE Region 10 Symposium, 99-104.

[13] Guo, C., et al. (2015) \$alpha-Fair Power Allocation in Spectrum-Sharing Networks. IEEE Transactions on Vehicular Technology, 1-1.

[14] Han, S., Zhang, X. and Shin, K. (2016) Fair and Efficient Coexistence of Heterogeneous Channel Widths in Next-Generation Wireless LANs. IEEE Transactions on Mobile Computing, 1-1.

[15] Asif, Z., Qadir, J. and Baig, A. (2013) Channel Assignment in Non-Cooperative Coexisting Co-Located Independent Cognitive Radio Networks. International Multi Topic Conference, 115-120. https://doi.org/10.1109/INMIC.2013.6731335

[16] Lim, J.G., Chou, C.T. and Jha, S. (2007) Non-Cooperative Coexistence of Co-Located Independent Wireless Mesh Networks. IEEE International Conference on Mobile Adhoc and Sensor Systems, IEEE, 1-9. https://doi.org/10.1109/MOBHOC.2007.4428645

[17] Tan, C.K., Sim, M.L. and Chuah, T.C. (2008) Game Theoretic Approach for Channel Assignment and Power Control with No-Internal-Regret Learning in Wireless Ad Hoc Networks. IET Communications, 2, 1159-1169. https://doi.org/10.1049/iet-com:20070547

[18] Lim, J.G., Chou, C.T. and Jha, S. (2009) Socially Conscious Channel Selection in 802.11 WLANs for Coexistence in a Non-Cooperative Environment. International Symposium on Modeling Analysis and Simulation of Wireless and Mobile Systems, MSWIM 2009, Tenerife, October 2009, 155-162.

https://doi.org/10.1145/1641804.1641832

[19] Gardellin, V., et al. (2011) G-PaMeLA: A Divide-and-Conquer Approach for Joint Channel Assignment and Routing in Multi-Radio Multi-Channel Wireless Mesh Networks. Journal of Parallel \& Distributed Computing, 71, 381-396. https://doi.org/10.1016/j.jpdc.2010.10.008

[20] Stüber, G.L. (2011) Principles of Mobile Communication. 
[21] IEEE (2010) P802.221.22TM/D0.1 Draft Standard for Wireless Regional Area Networks Part 22. Cognitive Wireless RAN Medium Access Control (MAC) and Physical Layer (PHY) Specifications: Policies and Procedures for Operation in the TV Bands, IEEE, 2006.

[22] Friedman, J.W. and Mezzetti, C. (2001) Learning in Games by Random Sampling. Journal of Economic Theory, 98, 55-84. https://doi.org/10.1006/jeth.2000.2694

[23] Monderer, D. and Shapley, L. Potential Games Games and Economic Behavior, 14.

[24] Gardellin, V., Das, S.K. and Lenzini, L. (2010) A Fully Distributed Game Theoretic Approach to Guarantee Self-Coexistence among WRANs. INFOCOM IEEE Conference on Computer Communications Workshops, 1-6. https://doi.org/10.1109/INFCOMW.2010.5466713

[25] Ishibuchi, H., et al. (1997) Fuzzy Q-Learning for a Multi-Player Non-Cooperative Repeated Game. Proceedings of the 6th IEEE International Conference on Fuzzy Systems, Barcelona, 1-5 July 1997, 3, 1573-1579.

https://doi.org/10.1109/FUZZY.1997.619776

\section{Submit or recommend next manuscript to SCIRP and we will provide best} service for you:

Accepting pre-submission inquiries through Email, Facebook, LinkedIn, Twitter, etc. A wide selection of journals (inclusive of 9 subjects, more than 200 journals)

Providing 24-hour high-quality service

User-friendly online submission system

Fair and swift peer-review system

Efficient typesetting and proofreading procedure

Display of the result of downloads and visits, as well as the number of cited articles

Maximum dissemination of your research work

Submit your manuscript at: http://papersubmission.scirp.org/

Or contact ijcns@scirp.org 